\title{
An Intelligent Segmentation System For Lateral Skull X-Ray Images.
}

\author{
D.N. Davis \& C.J. Taylor \\ Wolfson Image Analysis Unit \\ Department of Medical Biophysics \\ University of Manchester \\ Oxford Road \\ MANCHESTER M13 9PT
}

This paper describes research into knowledge-based image interpretation in clinical orthodontics. Similar work on knowledge-based methods is reviewed. We consider how a tiered knowledge-based system may provide an effective solution to the problems encountered. An autonomous intelligent segmentation system, for finding model-based features in lateral skull $x$-ray images, is described. Initial results are given.

The intelligent segmentation system reported here is part of a larger image processing tool that is being developed to automate the analysis of lateral skull x-ray images in clinical orthodontics. Although manual methods and interactive computer systems exist for performing this analysis (known as cephalometry), they tend to be unreliable in the placement of fiducial points. Consequently errors in clinically relevant measurements of distance and angle tend to ensue. An automated image interpretation system that could locate designated

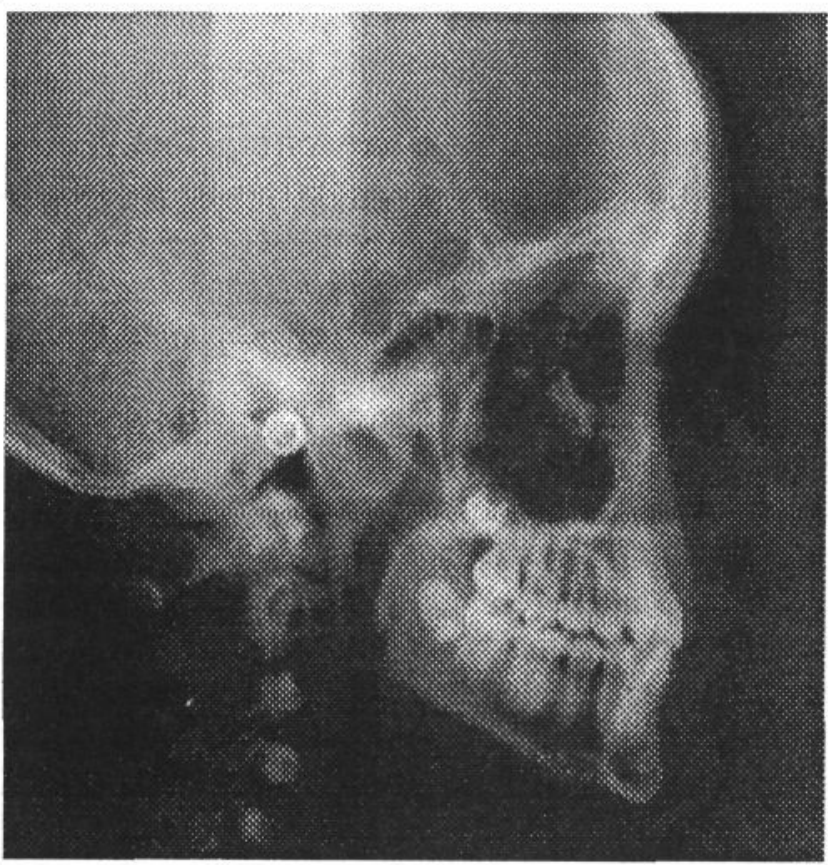

Figure 1. Digitised Lateral Skull x-ray

landmarks and perform a systematic analysis based on the found landmarks might solve this problem. In this paper we define the nature of the task and review previous attempts to automate the process. We provide the context for our current work by discussing the design of a complete system and the role of an intelligent segmentation sub-system (ISS). We then detail the ISS system, and present some initial results. Finally we discuss how the system could be improved.

\section{Background}

Figure 1 shows a typical digitised lateral skull $x$-ray. Figure 2 shows a limited number of landmarks placed on a manual tracing of figure 1 ; the most important

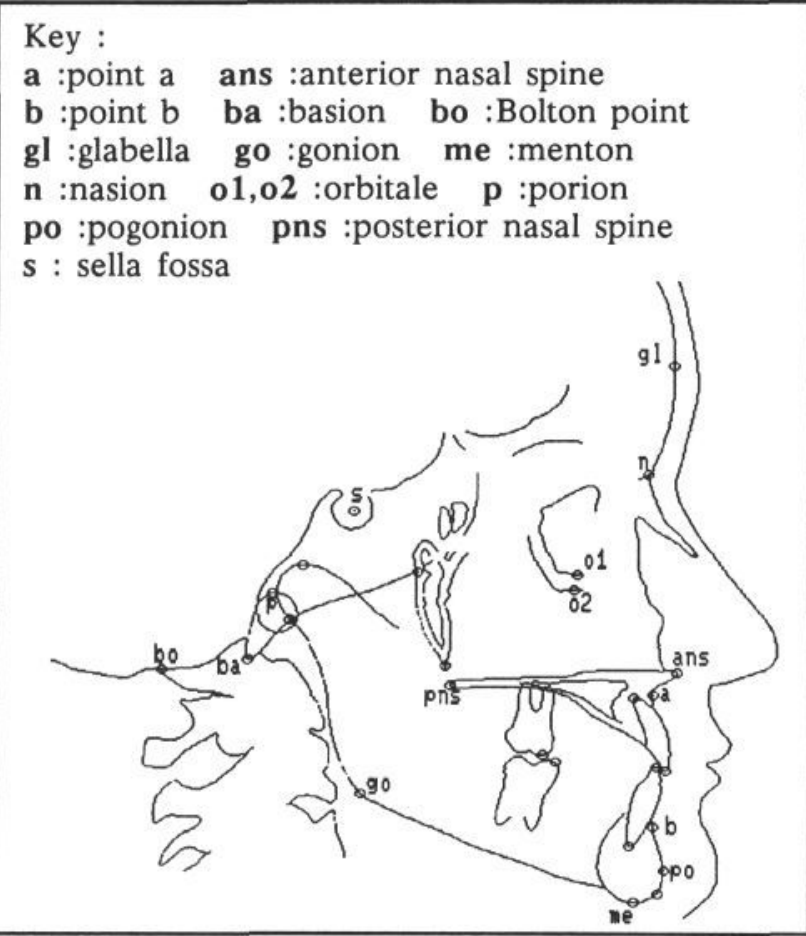

Figure 2. Manual Cephalometric Tracing

landmarks are named. These are used in cephalometric analyses for providing angular (eg s-n-a, s-n-b etc) and ratio measurements (eg n-a : n-b) that can be used to predict growth or help in deciding upon the nature of any necessary orthodontic treatment. Structures of interest can be manually traced and then used to locate landmarks, or the landmarks can be directly identified using interactive graphics. Most orthodontics departments use one, or both of these methods. The correct identification of the landmark points tends to be error prone, and varies between clinicians, resulting in 


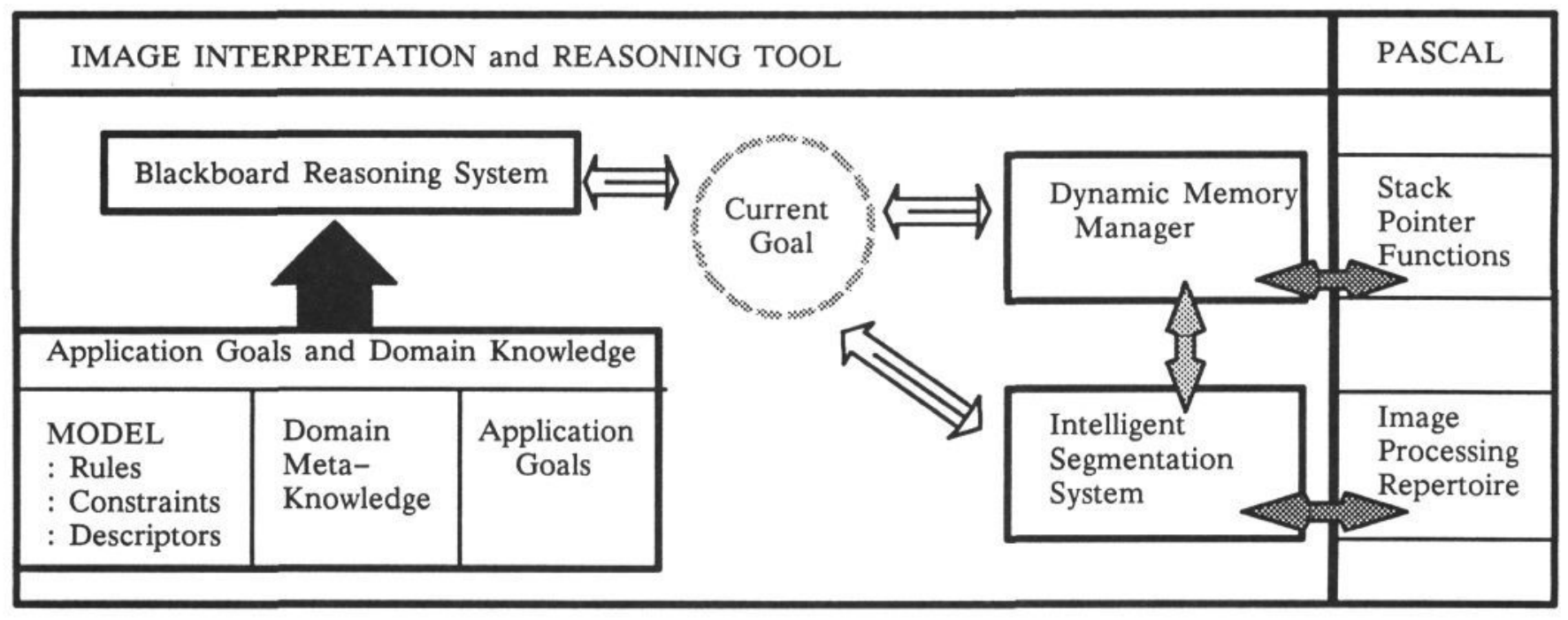

Figure 3. Schematic Representation of Image Reasoning Tool

inaccurate analyses. Furthermore a detailed analysis, covering over fifty points, can be time consuming. An automated analysis, using computer vision techniques, could provide more systematic and accurate results. Chen, Ip and Linney [2] conducted a preliminary study, using an imperative system allied to an array processor. This system was limited to the identification of only two landmarks, the sella fossa and the menton (see Figures 1,2), within interactively selected localised areas of the $\mathrm{x}$-ray film. A more ambitious project [7, 14] used an elementary knowledge-based system that would work only on relatively good quality $\mathrm{x}$-ray plates.

This latter system typifies a methodology which has often been applied to medical image interpretation. A high level module is used to model an application, so that particular, declaratively defined, features can be matched to the results of a rigidly defined low-level segmentation module. The segmentation module typically convolves a low-pass, or smoothed image, with a particular edge operator to produce a list of edge segments. Domain knowledge, usually represented in production rule format or structured set of frames, is then applied to the list of line segments. While such systems have achieved some measure of success $[6,10]$, the results are heavily dependent upon the preprocessing and edge detection processes. When the chosen operators fail, typically because assumptions about the nature of the image are ill founded [7], the whole system breaks down and acts in a quite unintelligent manner. It has been suggested [3, 4] that alternative modes of segmentation, involving low level knowledge-based modules may provide the adaptability required for medical image segmentation.

Some interesting solutions have been proposed for applying knowledge-based methods to image segmentation. Nazif and Levine [9] demonstrated that heuristics, typically used in imperative segmentation systems, can be expressed, to good effect, at the production rule level. Their system perhaps suffers from attempting to provide a too general solution, leading to ambiguities in image interpretation. The work of Smets and others [10], on subtraction angiography, provides more meaningful results, benefiting from a goal directed approach to image interpretation. A high level model-based system is used to interpret the results of a rule based segmentation system. The segmentation is constrained, by the nature of its knowledge source, to finding objects that fit with a most general model of blood vessels. Both these systems, and the IBIS system [3] which simply modifies segmentation parameters, are still limited by the available repertoire of image processing techniques. Meisels and Bergmann [8] have shown that a knowledge of image types, and alternative methods of processing a particular class of image are appropriate to systems that deal with images of varying quality. They did however restrict their system to straight line objects within aerial images.

\section{Overview Of The Image Reasoning Tool}

An automated cephalometric radiology system will need the capability for coping with objects of subtly varying morphology in images of varying quality. Our initial efforts showed that although some sequences of low level image processing provided cues to support the presence of a given feature, the variations in image quality, both digital and on the x-ray film, and in the shape and visual definition of the biological features sought, suggested a more adaptable approach to the problem would be required. A hierarchical model of the lateral view of the head could be developed, that would encompass object definitions and expected areas of interest. The principle function of a knowledge-based system that included such a model would be to reason about application goals, using evidence from the model and any given images, in order that these goals be satisfied. It is envisaged that at various stages in the chain of reasoning, evidence from the chosen source image will be required, so that hypotheses regarding the location of selected features in the source image can be 


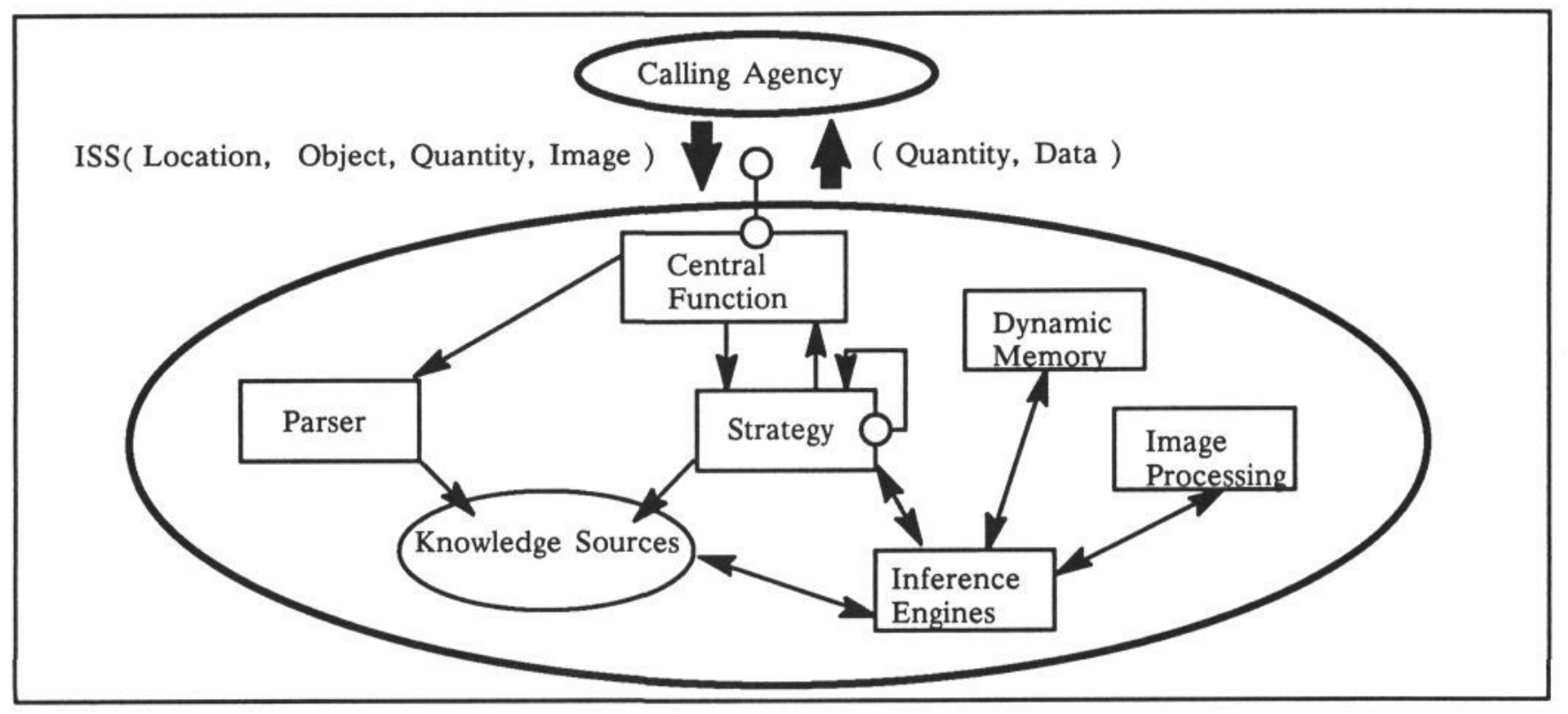

Figure 4. Schematic Representation of Intelligent Segmentation System

accepted or rejected. Further investigation demonstrated that factors beyond our control, for example the quality of the $\mathrm{x}$-ray plates and the diverse nature of the radiolucencies of some structures across patients, would require a repertoire of image processing techniques to be available if useful segmentation of an area of interest were to be feasible. It would seem plausible that the strategies and intelligent behaviour typified by artificial intelligence and knowledge-based systems, would be applicable to the decisions required to accomplish successful segmentation and locate the desired object where present.

The overall system we propose will have a blackboard architecture bearing similarities to those of Tsotsos [12], and Woods [13]. Where it will certainly differ from such systems is that it will not have direct access to the image, or the image analysis modules. Figure 3 depicts the architecture of such a blackboard system. A sophisticated hypothesise and test shell incorporating reasoning mechanisms, model handling facilities and a dynamic memory handling module would also include an autonomous intelligent segmentation system. A hierarchical model of the lateral skeletal head, together with a framework of application goals would be provided for this application. In order to test hypotheses regarding the location of objects within an image, the system would create goals, requiring image access via the intelligent segmentation system (ISS).

\section{Overview Of The Intelligent Segmentation Tool ISS}

We have designed and implemented an ISS to deal with the types of objects expected in lateral skull x-rays. The ISS, schematically represented in Figure 4, is a modular system with each module having clearly defined tasks. A central function initialises the system, calls further modules to parse the model of the desired object and then to find that object. The parser creates a net depicting relations between further objects, and a knowledge source defining further constraints on the set of objects. The strategy module works on the defined net finding objects referred to by nodes in the net. This is done be activating strategy cells that are responsible for finding different classes of object and image data. The strategy cells can access the object knowledge source and refer items to the inference engine. The inference engine includes forward and back chaining mechanisms. These can operate in cyclic, ie a rule may be fired any number of times, or in one-hit modes. In one-hit mode, a rule may only be used once for each call to the inference engine. The inference engine is the only module to access all knowledge sources, including the creation of short lived knowledge relating the success and failure of rule sequences in attempting to find the current object data. The inference engine also has full access to the image processing modules, and dynamic memory functions that are referenced in rules and assertions.

\section{Details Of The Intelligent Segmentation System}

For the cephalometric domain of application, it is possible to define suitable objects using only three object primitives, the open-curve, the closed-curve and the point, together with a set of combination types. Such an approach is consistent with a hierarchical model of the head where the base elements are a number of perceptual primitives. For instance, we can define the forehead as an obtuse convex open-curve, the nasal bone as an acute convex open-curve, and the nasion point as the point of the upper $\mathrm{T}$-junction of these two curves. The closed-curve object can be used to represent the boundary of a region, which may be further described as a constrained combination of open-curves. Associated with these primitive types are constraints which can be used to define a particular 
exemplar of that type. Points can be defined on other objects using combination types, eg $\mathrm{X}$-junction, T-junction, convextip etc. Complex objects may be defined using combination types and primitive objects; constraints being used to restrict the primitive object components and the nature of the relationship between the components.

The system is called by name with parameters defining a reference to an area of interest, the object to be found, a minimum quantity of that object, and a reference to a source image. Checks are made on the validity of these parameters. The object definition is passed to the parser unit, to be represented as hierarchical net. The net consists of nodes referencing a simple object, or a complex object encompassing the geometric and morphologic relations between further sub-objects. Each node references an object frame. An object frame is a default representation, which is updated with the constraints of a given object description. The net, object frame and frame slot identifiers are consistent with the terms allowed to define rules in the image processing repertoire knowledge source. When successfully parsed the object frames are placed on an object stream. The net is a global object that refers to items on the object stream. The central ISS function completes the initialisation of knowledge sources and calls the strategy unit.

Modules within the strategy unit can access the net, but all knowledge sources, or streams, are handled by the inference engines. The strategy unit attempts to satisfy the net by scheduling the finding of the nodes of the net using appropriate strategy cells. The strategy cells combine with the inference engine, within the strategy module to work as a goal directed hierarchal production system. The use of several strategy cells, including the multiple use of cells, allows for aggregation of evidence to satisfy complex constraints. The strategy cells make decisions upon the processing required by considering the results of the inference engine. Certain combinations of strategy cells can act as an error correction, or backtracking, mechanism where required. Although goal directed, the system demonstrates a preference to forward chain, using the assertion and rule streams. When finding a particular object, the strategy unit temporarily places the frame representing that object on the assertion stream. The result of firing a rule causes the assertion stream to be updated. This may result in data pertaining to an object to be permanently added to the assertions. Where forward chaining is not successful in producing the required segmentation and a successful object match cannot be found, the inference engine is used in single-hit backward chain mode. In this mode the unit chooses the best untried rule sequence to achieve its goals, backtracking where necessary. If no candidates for an object can be found, the strategy unit reports a fail, and the ISS central function acts appropriately, informing the user and calling system of this fact. If more than the required number of candidates are found, the system attempts to provide the best possible candidates.

The image processing repertoire is activated by the selection of the appropriate rules. The incorporated functions are not specific in their processing to cephalometric images. Our initial imperative work was of use in helping to define what forms of processing would produce the desired results, and what alternatives could be used where obvious choices failed. These alternative approaches make use of new and existing software available in the Wolfson Image Analysis Unit $[5,11,1]$. The repertoire of image processing techniques include median operators, canny operators, sobel operators, linear operators, enhancement processes, line tracking algorithms, shape matching strategies and intensity matching functions. A decision was made to keep all direct image access functions separate from the knowledge-based segmentation system, as distinct modules. The repertoire can be readily extended, requiring the module itself, appropriate rules in the processing knowledge source and an interface between the ISS and the module.

\section{Initial Results}

The ISS has been implemented in Sun Common LISP and tested from an interactive shell. Image processing modules are written in Pascal. Initial results have been obtained with $\mathrm{x}$-ray images of varying quality. The system can readily find simple objects, and table 1 gives the results for testing the system for five features over twenty images. Figure 5 shows these features, plus some fudicial points, successfully found on one image. The segmentation system succeeds in finding required objects because the various alternative processing techniques together provide supportive evidence for an

\begin{tabular}{|c|c|c|c|c|}
\hline Forehead & Symphysis & Supramentale & Cranial Base & Subspinale \\
\hline 19 & 18 & 13 & 17 & 18 \\
\hline
\end{tabular}

Table 1 ISS Successes

(for 5 features on 20 Images )

object. Only when an exhaustive search of alternative strategies provides no satisfactory match does the system return messages to the effect of no evidence to support this object within the given localised area. The above results are for an initial system. We hope to improve on this by extending the system in a number of ways. Where an object cannot be found and is present, the system typically fails for one of two reasons; either no match is made, or an incorrect match is made. The grey level edge segments produced may be of very poor quality. The extension of the ISS repertoire to include better edge detection methods, eg model driven linear operators, may help. Secondly the system does not currently combine edge segments. The inclusion of explicit line joining heuristics within the ISS knowledge 
base, in a manner similar to Nazif and Levine [9], should prove to be of considerable benefit. These heuristics should include the capabilities to join line segments based on the results of appropriate linear grey level operators. An improvement in feature to model matching may result from a more sophisticated measure of match, or a greater aggregation of feature attributes within the models. At present the system uses a normalised difference from model measure, with a variable exclusion level. This ought to be changed to a trapezium probability measure.

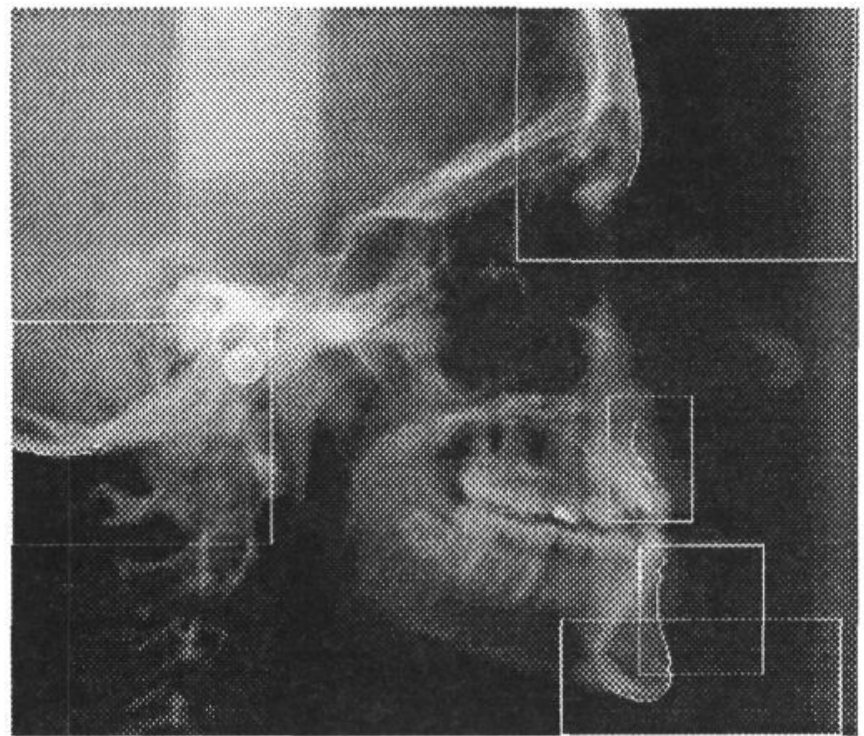

Figure 5. Features found using the ISS

At present the strategies used to discover relevant aspects of objects are encompassed in a set of hierarchal functions. To make the system more general, these need to be held as a strategy knowledge source. It would then allow the inference engine to direct its actions when dealing with image processing knowledge sources. The system has yet to make full use of found primitives in finding other more difficult elements of complex objects. While such a system is intended for cephalometric $\mathrm{x}$-ray use, it may be applicable to a much wider domain. The implementation of the top level image reasoning tool would allow for a completely automatic cephalometric analysis.

\section{Acknowledgements}

This work is supported by a SERC research studentship and a North West Regional Health Authority project grant. We thank Professor W.C. Shaw and Dr. F. MacKay, of the Department of Orthodontics, for their assistance on this project.

\section{References}

1. Azzopardi P.J., Pycock D., Taylor C.J. \& Wareham A.C. 'An experiment in model-based boundary detection.' Proceedings AVC88 (1988) pp31-36.

2. Cohen A.M., Ip G.C. \& Linney A.D. 'A preliminary study of computer recognition and identification of skeletal landmarks as a new method of cephalometric analy- sis.' British Journal of Orthodontics, Vol. 11 No. 3 (1984) pp134-154

3. Dellepiane S., Serpico S.B., Venzano L. \& Vernazza G. 'Structural analysis in medical imaging.' Proceedings Seventh Conference on Electrotechnics: Advanced Technologies and Processes in Communications and Power Systems Paris (1986) pp 522-529.

4. Garbay C. 'KISS: A knowledge-based image segmentation system.' Proceedings of the Ninth International Conference on Pattern Recognition, Beijing, China, (1988).

5. Graham J. \& Taylor C.J. 'Boundary cue operators for model based image processing.' Proceedings AVC 88 (1988) pp 59-64.

6. Lebel O. 'ARCHI: An expert system for biological objects recognition.' Pattern Recognition Letters, Vol. 8 No.2 (1988) pp 131-139.

7. Levy-Mandell A.D., Venetsanopoulos A.N. \& Tsotsos J.K. 'Knowledge-based landmarking of cephalograms.' Computers \& Biomedical Research, Vol. 19 (1986) pp 282-309.

8. Meisels A. \& Bergman S. 'Finding objects on aerial photographs: A rule-based low level system.' Proceedings of CVPR'88, June 5-9, 1988, Ann Arbour, Michigan USA. (1988) pp 118-122

9. Nazif A.M. \& Levine M.D. 'Low level image segmentation: An expert system.' IEEE Transactions on Pattern Analysis and Machine Intelligence, PAMI-6(5) (1984) pp 555-577.

10. Smets C., Verbeeck G., Suetens P. \& Oosterlinck A. 'A knowledge-based system for the delineation of blood vessels on subtraction angiograms.' Pattern Recognition Letters, Vol. 8 No. 2 (1988) pp 113-121.

11. Thornham A., Taylor C.J. \& Cooper D.H. 'Object cues for model based image interpretation.' Proceedings AVC88 (1988) pp 53-58.

12. Tsotsos J.K. 'Representational axes and temporal cooperative processes.' Technical Report RCBVTR-84-2. The University of Toronto, (1984).

13. Woods P.W., Pycock D. \& Taylor C.J. 'A frame-based system for modelling and executing visual tasks.' Proceedings AVC88 (1988) pp 45-51.

14. Yan C.K., Venetsanopoulos A.N. \& Filleray E.D. 'An expert system for landmarking of cephalograms.' Proceedings of the 6th. International Workshop on Expert Systems \& Applications (1986) pp 337-356. 
